\title{
Life-threatening neurological syndrome in Down's syndrome
}

\author{
Martino Ruggieri, Vito Pavone, Agata Polizzi, Enrico Parano, Alessandra Tinè
}

This 19-year-old girl was the full-term product of an uncomplicated pregnancy and normal delivery, diagnosed at birth as having Down's syndrome (karyotype: $47,+21$ ). Developmental milestones were mildly delayed, she had characteristic phenotype, an uncorrected atrial septal defect, and mild mental retardation. She showed excellent sitting balance and walked with both hands held high. At age seven years, routine blood tests and screening $\mathrm{X}$-rays were unremarkable. At age nine years, due to her progressing hypotonia, she was enrolled in a special school that emphasized head, neck and trunk patterning. Within a year after enrolment, progressive neck pain, increased fatiguability on walking, and impaired gait were noted.

Neurological examination at the age of 10 years revealed lower limb weakness, patellar hyperreflexia, up-going toes, calcanear clonus, impaired gait with spasticity and proneness to fall, and gross motor uncoordination. Routine blood tests were normal; neck radiography showed an anterior arch-odontoid distance ranging from $>6.0 \mathrm{~mm}$ in neutral position to $12 \mathrm{~mm}$ during flexion. Magnetic resonance imaging (MRI) of the brain and spinal cord (figure) was carried out. After this diagnostic procedure the patient's family declined treatment because of ongoing complex medical problems, including recurrent pneumonia and insulin-dependent diabetes mellitus.

University of Catania, Catania, I-95125 Italy Division of Paediatric Neurology, Paediatric Clinic

M Ruggieri

A Polizzi

E Parano

$A$ Tinè

Orthopaedic Clinic

V Pavone

Department of Clinical
Genetics, Oxford
Radcliffe Hospital
NHS Trust, Oxford
OX3 7LJ, UK
M Ruggieri

Within two years there was increased loss of ambulation and of bowel and bladder function; progressive spasticity, weakness and neuromuscular scoliosis ensued. She was confined to

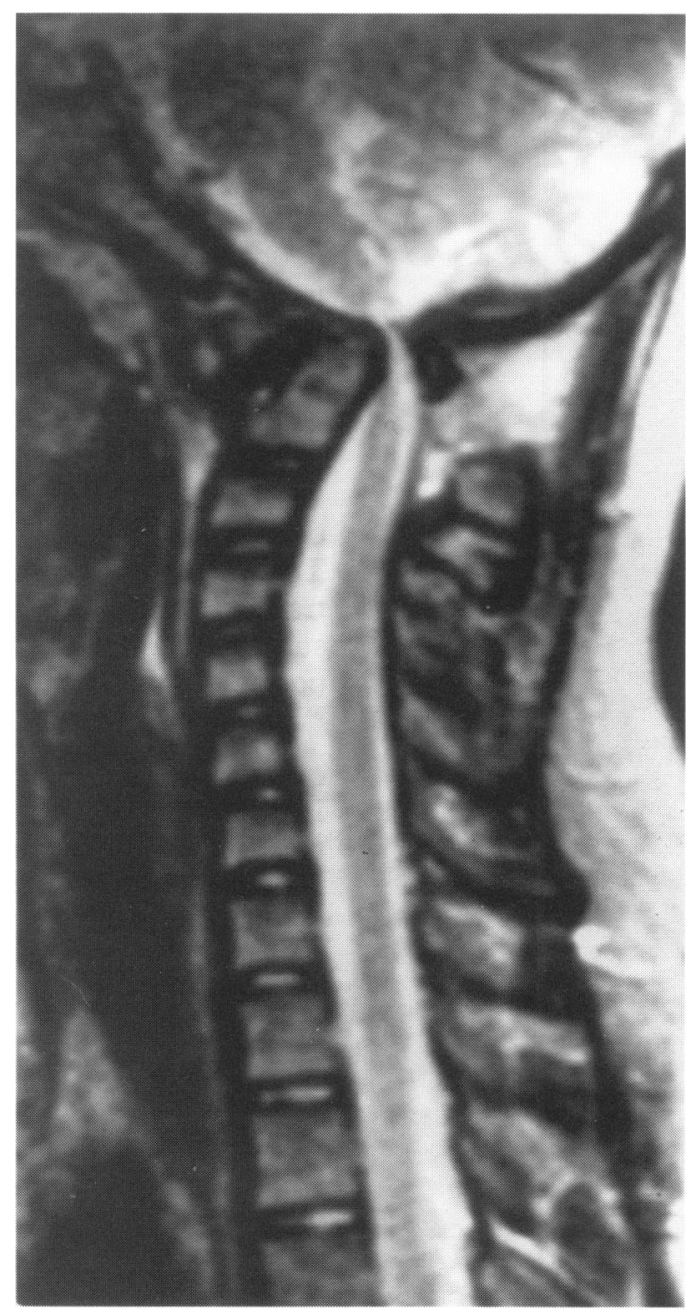

Figure T1-weighted sagittal MRI scan of patient at age 10 years a wheelchair.

The girl, who is currently 19 years old, has moderate mental retardation (IQ $=64)$, respiratory failure, increased muscle tone in all four extremities, severe scoliosis and upper and lower limb contractures, weakness (grade 4/5), hyperreflexia with bilateral ankle clonus, bilateral extensor plantar responses and sensory level. She is confined to bed and bowel and bladder incontinent. There are no seizures. Her parents still refuse consent for treatment due to the expected poor outcome.

\section{Questions}

1 What is the lesion shown on MRI and what is the most likely cause of the MRI appearance ?

2 What was the final diagnosis and what are the prognostic implications?

3 What was the treatment advised?

4 What intervention would you recommend to prevent this neurological syndrome in patients with Down's syndrome? 
Answers

QUESTION 1

There is evidence of marked narrowing of the cervical spinal cord at the level of $\mathrm{C} 1 / \mathrm{C} 2$ and atlantoaxial dislocation. In patients with Down's syndrome, congenital hypotonia and laxity of the transverse ligament of $\mathrm{C} 1$ or dysplasias of $\mathrm{C} 1 / \mathrm{C} 2$ can predispose to dislocation/subluxation of the atlantoaxial joint with potential neurological sequelae. ${ }^{12}$

QUESTION 2

The final diagnosis was atlantoaxial dislocation with acute symptomatic compression to the spinal cord secondary to physical therapy procedures in a child with Down's syndrome and atlantoaxial instability (AAI). The prognosis is poor and, although a few patients remain stable for months to years, there is occasional progression of symptoms, eventually leading (as in the present case), to paraplegia, hemiplegia, or quadriplegia, with respiratory failure or death.

QUESTION 3

Treatment options include decompression by traction and stabilisation of the cervical subluxation by rewiring of the $\mathrm{C} 1$ ring and posterior spinal fusion. ${ }^{23}$

\section{QUESTION 4}

Even though often clinically silent, AAI is the rule in children with Down's syndrome. ${ }^{12}$ Most dislocations occur in the course of everyday activity, medical treatment or coincidentally whilst participating in sport. Any medical, operative, physical and rehabilitative procedure in these patients should be preceded by a careful search for any pre-existing warning signs and symptoms of cervical cord compression. A high degree of suspicion is necessary because subtle symptoms may be attributed to other disease processes. The evaluation of these symptoms in children with Down's syndrome is also more difficult due to the marked delays that may occur in motor and developmental milestones. From the available scientific evidence, it is reasonable to conclude that lateral plain radiographs of the cervical spine are of potential but unproved value in detecting patients at risk for developing spinal cord compression. $^{23}$ Thence, recognition of symptomatic patients is still challenging and requires frequent interval histories and physical examinations, including evaluations before undergoing medical or physical procedures or participating in sports, preferably by physicians who have cared for these patients for a while. Eliciting a history of abnormal head posture, restricted neck movement and/or pain, altered gait, or regression from previously attained skills in ambulation or toilet training, for instance, could be important clues. Any child with such symptoms should be referred to a specialist orthopaedic or neurosurgeon for further investigation. ${ }^{2}$
Cervical spine instability in Down's syndrome

- hypotonia, ligamentous laxity or dysplasia of the atlantoaxial joint allow atlantoaxial dislocation/subluxation leading to cervical cord compression and severe neurologic damage

- even though atlantoaxial instability (AAI) is the rule, it is very often clinically silent

- many patients with symptomatic AAI may have symptoms and signs of cervical cord compression for weeks or years before they are recognised as having neurological disease

- these signs and symptoms include abnormal head posture, restricted neck movement and/or pain, altered gait, regression from previously attained motor skills or skills in toilet training

- medical, operative, physical or rehabilitative procedures should be preceded by a careful search for any pre-existing sign or symptom of spinal cord compression

\section{Discussion}

The first report of AAI in persons with Down's syndrome and neurologic consequences was published by Dzenitis in $1966 .{ }^{4} \mathrm{He}$ described a 13-year-old girl with Down's syndrome in whom torticollis and increasing neurologic signs developed after an episode of otitis media. Since that time, a total of 41 reasonably complete case reports in the paediatric age group ( $<21$ years), have been published ( 31 girls and 10 boys). ${ }^{3}$ Six additional individuals have been described in the literature but with incomplete information. ${ }^{3}$

The neurological manifestations include easy fatiguability on walking, abnormal gait, neck pain, limited neck mobility, torticollis or head tilt, poor co-ordination and clumsiness, sensory deficits, spasticity, hyperreflexia, clonus, extensor plantar reflex, deterioration in bladder or bowel control and other upper motor neuron and posterior column signs and symptoms. ${ }^{3}$ Such symptomatology often remains stable for months or years; occasionally it progresses, with potentially catastrophic sequelae. Thirteen of the 41 reported children with symptomatic AAI had trauma before symptoms first appeared ${ }^{3}$ : namely, four became symptomatic after a fall or a tumbling accident, two during physical activity concerning sport, six after medical procedures such as general anaesthesia for cardiac surgery, appendicitis, eye surgery, liver biopsy or after rehabilitative procedures, and one patient became quadriplegic after a motor vehicle accident. Two of the 41 reported patients with symptomatic AAI gradually worsened their previous neurological symptoms after a fall and a trampoline accident, respectively. ${ }^{3}$ Of the remaining 26 patients, one had relapsing neurological symptoms after a fall, and in the others causative or precipitating events were difficult to determine or apparently followed upper respiratory tract infections or even were associated with other (osteoarticular) diseases. ${ }^{3}$ Only one case, ${ }^{5}$ of the six who became symptomatic after medical procedures, ${ }^{3}$ was referred to hospital because of concern about neuromotor deterioration 
after a rehabilitative procedure. This was a 5-year-old girl with excellent sitting balance and discrete walking autonomy who had severe upper extremity tremor and spasticity one year after a vigorous programme of neck and head exercise. She became hemiparetic after sedation for neurological studies (electroencephalogram and neuroimaging). She partially recovered after surgical stabilisation (Gallie fusion).

Our patient had progressive neuromotor degeneration after 12 months of an intensive programme of neck, head and trunk exercise; her symptomatology, which rapidly progressed, confining her to a wheelchair and finally to bed, was almost certainly triggered by physical therapy procedures, as a radiograph obtained before her enrolment in the special school showed a normal atlantoaxial gap in all neck positions. Several studies have demonstrated that radiological status can change over time because of variations in atlantoaxial instability.

1 Morton RE, Ali Khan M, Murray-Leslie C, Elliott S. Atlantoaxial instability in Down's syndrome: a five year follow-up study. Arch Dis Child 1995;72:115-9.

2 Marder E, Dennis J. Medical management of children with Down's syndrome. Curr Pediatrics 1997;7:1-7.

3 Committee on Sports Medicine and Fitness. Atlantoaxia instability in Down syndrome: subject review. Pediatrics instability in Dov
The aforementioned case reports ${ }^{35}$ indicate that many patients with symptomatic AAI may have symptoms and signs of cervical spinal cord compression for weeks or years before they are recognised as having neurologic disease. Current evidence suggests that these neurological abnormalities may be more predictive of potential progressive injuries than the abnormalities or radiography themselves in asymptomatic patients.

\section{Final diagnosis}

Atlantoaxial dislocation with acute symptomatic compression to the spinal cord secondary to physical therapy procedures in a child with Down's syndrome and atlantoaxial instability.

Keywords: Down's syndrome; atlantoaxial instability; spinal cord compression; neurologic complications; rehabilitative procedures

4 Dzenitis AJ. Spontaneous atlanto-axial dislocation in mongoloid child with spinal cord compression. 7 Neurosurg 1966;25:458-60.

5 Msall ME, Reese ME, Di Gaudio K, Griswold K, Granger $\mathrm{CV}$, Cooke RE. Symptomatic atlantoaxial instability associated with medical and rehabilitative procedures in children with Down syndrome. Pediatrics 1990;85:447-9.

\title{
Fever, thrombocytopenia, and diarrhoea
}

\author{
R B S Laing, A R MacKenzie, C C Smith
}

A 40-year-old man was admitted to hospital with a 10-day history of fever, malaise, profuse diarrhoea and intermittent macular rash affecting the chest and legs. On arrival, he had a fever of $39.5^{\circ} \mathrm{C}$, blood pressure $100 / 70 \mathrm{mmHg}$ and pulse rate 110 beats $/ \mathrm{min}$. There was no lymphadenopathy. The abdomen was tender but not distended and the rectum empty and non-tender Admission blood count revealed haemoglobin $9.8 \mathrm{~g} / \mathrm{dl}$, total white cell count $1.4 \times 10^{9} / 1$ and platelets $74 \times 10^{9} / 1$. The rectum appeared inflamed at sigmoidoscopy and rectal biopsy revealed a mild inflammatory infiltrate. Barium enema was unremarkable. The patient was treated empirically for bacterial gastroenteritis with intravenous ciprofloxacin but a series of stool and blood cultures were negative and over the next 10 days he continued to have a high swinging fever and between 10 and 20 loose stools daily. Empirical steroid therapy (prednisolone $30 \mathrm{mg} /$ day) was followed by a gradual improvement in his condition and at the time of discharge the diarrhoea had lessened and his haemoglobin was $10.5 \mathrm{~g} / \mathrm{dl}$, total white cell count $4.8 \times 10^{9} / 1$ and platelets $239 \times$ $10^{9} / 1$. When reviewed three months later he was taking $10 \mathrm{mg}$ prednisolone daily but his weight had fallen by $12 \mathrm{~kg}$ with intermittent severe diarrhoea. Increased steroid dosage did not alter his symptoms. Colonoscopy revealed no abnormality and colonic biopsies were normal. Serial stool cultures were again negative and microscopy revealed no ova, cysts or parasites. Blood count showed a haemoglobin of $11.9 \mathrm{~g} / \mathrm{dl}$, white cell count $3.9 \times 10^{9} / 1$ and platelets $63 \times 10^{9} / 1$.

\section{The Infection Unit Aberdeen Royal Infirmary, Aberdeen, Scotland AB25 2ZB, UK \\ R B S Laing \\ A R MacKenzie \\ C C Smith}

Accepted 24 October 1997

\section{Questions}

1 What acute infection might explain the thrombocytopenia and diarrhoea?

2 Which investigations would confirm the diagnosis?

3 What treatment should be offered? 